\title{
A quantitative analysis of the dispersion behavior of reduced graphene oxide in solvents
}

M. Ayán-Varela, J.I. Paredes, S. Villar-Rodil", R. Rozada, A. Martínez-Alonso, J.M.D. Tascón Instituto Nacional del Carbón, INCAR-CSIC, Apartado 73, 33080 Oviedo, Spain

\begin{abstract}
Detailed knowledge of the dispersion behavior of reduced graphene oxide (RGO) in solvents is important for its practical applications. Such behavior is expected to be different to that observed for pristine graphene, as a result of the chemically heterogeneous structure of RGO (patchwork of pristine and highly oxidized graphene domains). We have investigated the dispersibility of RGO in a wide range of solvents and analyzed the results on the basis of solvent surface energies and Hansen solubility parameters. Although RGO exhibited some features that are characteristic of pristine graphene, its dispersion behavior was dominated by its oxidized graphene domains, with alcohols being commonly the most successful solvents. Estimates of the effective Hansen parameters for RGO derived from the experimental data $\left(\delta_{\mathrm{D}} \approx 16.9 \mathrm{MPa}^{1 / 2}, \delta_{\mathrm{P}} \approx\right.$ 10.7 $\mathrm{MPa}^{1 / 2}$ and $\delta_{\mathrm{H}} \approx 14.1 \mathrm{MPa}^{1 / 2}$ ) were consistent with this view and afforded the rational design of solvent mixtures that surpassed the best single-component solvents in terms of dispersed amount of RGO. RGO-polymer composites could then be readily prepared in the best performing solvents. Overall, the present results provide a guide to the processing of RGO in the liquid phase with practical utility in the preparation of different graphene-based materials.
\end{abstract}

\section{Introduction}

As a novel two-dimensional material that exhibits a number of unique physical properties and a strong potential for application in many relevant areas, graphene is currently the focus of intensive research efforts worldwide [1-3]. A considerable fraction of such efforts are being devoted to the development of cost-effective and

*Corresponding author. Tel: (+34) 985119090 E-mail: silvia@incar.csic.es 
reliable methods that can afford the production of graphene in substantial amounts, which is a prerequisite for its widespread use in real-life applications [3,4]. In this regard, top-down production methodologies have received significant attention. These are mainly based on the bulk processing of graphite and graphite derivatives (e.g, graphite oxide) to give large quantities of graphene sheets, whether in pristine or chemically modified form, most notably as reduced graphene oxide (RGO) [4-7]. A particularly attractive feature of such methods is that they readily lend themselves to the preparation of graphene dispersions in the liquid phase [5-7]. Having access to graphene in colloidal dispersion is advantageous for a number of reasons. For instance, the dispersions can be processed into different macroscopic materials, such as thin films, coatings or composites, using straightforward techniques (e.g., spin- or spray-coating, solution blending, etc), and the properties of the graphene sheets can be tuned through various wet chemical functionalization schemes [6-9].

Previous studies have established that both pristine graphene and RGO sheets can be colloidally stabilized in aqueous and organic media with the assistance of proper dispersants (surfactants, polymers, biomolecules, etc.) [7,10-15]. Although these dispersions can be useful in some areas of application (for instance, biomedical research) [16], earlier work with other carbon nanostructures (e.g., carbon nanotubes) indicates that in many cases the presence of the dispersant, which is difficult to remove completely, can degrade the performance of the resulting materials or devices and is therefore undesirable [17]. Alternatively, certain solvents are capable in themselves of stably sustaining significant concentrations of graphene sheets, making such dispersantfree suspensions highly valuable towards many purposes. In the case of pristine graphene, and based on energetic considerations, Coleman and co-workers have theoretically predicted and then experimentally demonstrated that stable colloidal dispersions are favored in solvents with surface energies matching or close to that of the basal plane of graphite $\left(\sim 70-80 \mathrm{~mJ} \mathrm{~m}^{-2}\right)$ [5]. The observed dispersion behavior could also be rationalized on the basis of the cohesive energy density of the solvents, i.e. using their Hildebrand and Hansen solubility parameters, successful solvents possessing solubility parameters within a narrow range of values [18]. Both structurally and chemically, the surface of a pristine graphene sheet is homogeneous down to the atomic scale and essentially identical to the graphite basal plane. It is therefore not surprising that its interaction with solvent molecules, which governs the dispersibility of the sheets 
in the solvent, can be related to a parameter that roughly describes the propensity of the graphite basal plane to interact with other species (i.e., its surface energy).

On the other hand, RGO sheets are well known to display a locally heterogeneous structure, comprised of essentially pristine graphene domains a few nanometers in lateral size interspersed with heavily oxidized regions, where the residual oxygen functional groups characteristic of RGO are concentrated [19-21]. Compared to the case of pristine, oxygen-free graphene sheets, this patchwork-like structure of chemically differentiated domains can be expected to introduce some level of complexity in the analysis of the dispersion behavior of RGO in solvents. For example, a certain solvent might exhibit an optimal interaction with the pristine graphene domains of RGO and at the same time a poor or unsatisfactory interaction with its oxidized regions. While the formation of stable colloidal dispersions would be clearly favored in that solvent for pristine graphene sheets, it is far from obvious whether a similar trend would be observed when RGO sheets are used instead. Thus, from a fundamental science perspective such an issue is worth investigating. Although the dispersion behavior of RGO has already been qualitatively assessed in several solvents [22-24], to the best of our knowledge comprehensive and more quantitative studies, in the vein of those available for pristine graphene, have been lacking. Likewise, access to this type of information is useful from a practical point of view. Over the last years, RGO has become increasingly relevant in many prospective application areas [8,25-27], so a better knowledge of its dispersibility in solvents should facilitate its processing into valuable materials and devices.

Hence, in the present work we have investigated the dispersion behavior of RGO in a wide range of solvents, and the results have been quantitatively evaluated in the context of their surface energy and Hildebrand/Hansen solubility parameters. As could be expected from the above considerations, the dispersibility of RGO exhibited significant differences compared to that of pristine graphene, which could be rationalized on the basis of the structural peculiarities of the former. Significantly, this new insight allowed the formulation of solvent mixtures that exhibited enhanced efficiency in the dispersion and colloidal stabilization of RGO sheets. Likewise, concentrated suspensions in the best solvents were attained, which could be used for the preparation of RGO-polymer composites, as exemplified here with ethyl cellulose.

\section{Experimental}


The dispersion studies were carried out using RGO samples obtained by chemical reduction of aqueous graphene oxide (GO) suspensions. The solvents, starting graphite powder and other reagents were obtained from Sigma-Aldrich. First, graphite oxide was prepared from natural graphite powder through the Hummers method as reported elsewhere [28]. After purification with $1 \% \mathrm{HCl}$ solution and copious amounts of deionized water, the oxidized product was added to a given volume of water, subjected to bath sonication (JP Selecta Ultrasons system, $40 \mathrm{kHz}$ ) for $4 \mathrm{~h}$ and finally centrifuged (Eppendorf 5424 microcentrifuge) at $10000 \mathrm{~g}$ for $10 \mathrm{~min}$. The resulting supernatant, which was a suspension of exfoliated graphite oxide sheets (i.e., GO sheets), was collected for further use and its concentration estimated by means of UV-vis absorption spectroscopy using a previously described procedure [29]. As a particularly efficient and general-purpose reductant for $\mathrm{GO}$, hydrazine monohydrate was employed to convert the as-obtained sheets to RGO. More specifically, and following optimized methodologies [30,31], reduction was accomplished by heating aqueous GO dispersions $\left(3 \mathrm{mg} \mathrm{mL}{ }^{-1}\right)$ at $95^{\circ} \mathrm{C}$ for $1 \mathrm{~h}$ in the presence of hydrazine monohydrate $(64.5 \mathrm{mM})$ and ammonia $(44.6 \mathrm{mM})$. At such relatively high concentration of $\mathrm{GO}$, the dispersions tended to fully precipitate in a matter of minutes after completion of the reduction reaction. Note that if reduction was carried out in the absence of ammonia, precipitation would occur before completion of reaction and thus the final product would not be well reduced. The precipitated RGO product was thoroughly washed with deionized water and finally stored as aqueous slurry $\left(\sim 3 \mathrm{mg} \mathrm{mL}^{-1}\right)$ for subsequent use in the solvent dispersion tests.

For the dispersion tests, a given volume of the RGO slurry was diluted in the corresponding solvent by a factor of 30 (giving a starting concentration of RGO of about $0.1 \mathrm{mg} \mathrm{mL}^{-1}$ ), which was then bath-sonicated for $2 \mathrm{~h}$ and finally centrifuged for 5 $\min$ at different centrifugal forces $(500,1000$ or $2000 \mathrm{~g}$ ). In principle, working with a dried product rather than the wet slurry should also be possible. Control experiments with some selected solvents indicated that this was indeed the case. However, the RGO sheets in the dried product were heavily agglomerated, hampering their exfoliation and dispersion during the sonication step and thereby decreasing significantly the amount of material that could eventually be suspended in the solvents. On the other hand, in the wet state the RGO sheets were found to be in a much less aggregated conformation, which facilitated their detachment and transfer to the solvent medium. We note, however, that the relative ability of solvents to disperse any given material is not 
expected to depend on its initial aggregation state, provided that comparisons between solvents are made on an equal footing (i.e., using starting materials with the same aggregation state for all solvents) [32]. Therefore, for the sake of convenience we used the aqueous RGO slurry as starting material for our dispersion studies, but the overall results should be generally applicable to RGO in any other initial aggregation state. We also note that a marginal amount of water (volume fraction $\sim 3 \%$ ) will remain in all the dispersions. However, this will have little impact in our analysis, as we will explain below.

The amount of RGO retained in the supernatant after sonication and centrifugation was taken as a relative measure of the ability of the solvent to colloidally stabilize this material. Such an amount was estimated by determining the absorbance of the suspension at a wavelength of $660 \mathrm{~nm}$. Although RGO is known to exhibit a welldefined absorption peak at $\sim 268-270 \mathrm{~nm}$ (provided that the sheets have been well reduced) [30,31], the latter wavelength was not used in the present measurements. This is because many solvents (particularly those containing $\mathrm{C}=\mathrm{C}$ or $\mathrm{C}=\mathrm{O}$ structures) displayed very strong absorbance in the 200-300 nm wavelength range, which could not be compensated for in our double-beam spectrophotometer. However, all the solvents were completely transparent at wavelengths above $400 \mathrm{~nm}$. Thus, any absorbance measured at a given wavelength above $400 \mathrm{~nm}$ (e.g., at $660 \mathrm{~nm}$ ) could be safely related to the amount of dispersed RGO according to the Beer-Lambert law: $A / l=\alpha \mathrm{C}$, where $A$ is the measured absorbance, $l$ is the optical path length, $\alpha$ is the extinction coefficient and $\mathrm{C}$ is the concentration of RGO. To obtain meaningful sets of data, the dispersion experiments were carried out five times for each solvent, so that average and standard deviation values are provided.

Characterization of the samples was accomplished by means of UV-vis absorption spectroscopy, X-ray photoelectron spectroscopy (XPS), atomic force microscopy (AFM) and thermogravimetric analysis (TGA). UV-vis absorption spectra were recorded on a double-beam He $\lambda$ ios $\alpha$ spectrophotometer, from Thermo Spectronic. XPS data were obtained on a SPECS apparatus using a monochromatic Al K $\alpha$ X-ray source $(1486.3 \mathrm{eV}, 100 \mathrm{~W})$ and working at a pressure of $10^{-7} \mathrm{~Pa}$. Specimens for XPS were prepared by depositing the RGO dispersion drop-wise onto a metallic sample-holder pre-heated at $\sim 50-60{ }^{\circ} \mathrm{C}$ until it became uniformly covered by a dark film. AFM images of the RGO sheets were acquired with a Nanoscope IIIa Multimode instrument working 
in the tapping mode of operation. To that effect, rectangular Si cantilevers with resonance frequencies around $250-300 \mathrm{kHz}$ and spring constants $\sim 40 \mathrm{~N} \mathrm{~m}^{-1}$ were employed. In this case, a small volume $(\sim 10-20 \mu \mathrm{L})$ of diluted RGO dispersion ( 0.01$\left.0.02 \mathrm{mg} \mathrm{mL}^{-1}\right)$ was drop-cast onto a pre-heated $\left(\sim 50-60^{\circ} \mathrm{C}\right)$ highly oriented pyrolytic graphite (HOPG) substrate and allowed to dry. TGA was accomplished with an SDT Q600 thermobalance (TA Instruments) under synthetic air gas flow (100 $\mathrm{mL} \mathrm{min}^{-1}$ ) at a heating rate of $10^{\circ} \mathrm{C} \mathrm{min}^{-1}$, using platinum crucibles.

\section{Results and discussion}

Fig. 1a (left vial) shows a digital photograph of the starting aqueous GO dispersion diluted by a factor of 30 (to about $0.1 \mathrm{mg} \mathrm{mL}^{-1}$ ), so that the yellow-brown tone characteristic of as-prepared GO can be clearly appreciated. Following reduction with hydrazine monohydrate in the presence of ammonia, the color of the dispersion changed to opaque black (Fig. 1a, right vial), indicating successful conversion of the GO sheets to RGO [29,30]. This observation was confirmed by UV-vis absorption spectroscopy, as revealed in Fig. 1b. The spectrum of unreduced GO (orange plot) was dominated by an absorption peak located at $\sim 231 \mathrm{~nm}$, which has been ascribed to $\pi \rightarrow \pi^{*}$ transitions from the small-sized electronically conjugated domains present in this material $[30,33]$. Removal of a fraction of the oxygen functional groups from the sheets as a result of reduction tends to increase the size of these domains [20,34], which in turn is reflected in a red-shift of the absorption peak position [31]. For the RGO sheets prepared here, the absorption peak red-shifted to about $268 \mathrm{~nm}$ (black plot), indicative of well-reduced samples [30,31]. XPS provided more direct evidence of successful reduction: survey spectra (not shown) indicated that the $\mathrm{O} / \mathrm{C}$ atomic ratio for the RGO samples was 0.08 , compared with a value of 0.43 for their unreduced counterpart. In addition, the high resolution $\mathrm{C} 1 \mathrm{~s}$ spectrum of unreduced GO (Fig. 1c, orange plot) showed three components, centered at 284.6 (non-oxidized carbon atoms in graphitic environments), 286.6 (oxidized carbon atoms in C-O configurations), and $287.9 \mathrm{eV}$ (oxidized carbon atoms in $\mathrm{C}=\mathrm{O}$ configurations) [29]. By contrast, the relative contribution of the oxidized carbon atoms decreased considerably after reduction (Fig. 1c, black plot), demonstrating that the number of carbon atoms in an oxidized state was largely diminished. There is also some nitrogen present in $\mathrm{RGO}$, the N/C atomic ratio being 0.02. As shown in the AFM image of Fig. 1d, the obtained RGO product was formed by single-layer sheets with lateral dimensions typically between a few and several hundred nanometers. This 
RGO material, which was obtained as a precipitate, was extensively washed with deionized water and kept as aqueous slurry for use in the dispersion tests.

The dispersibility of a given solute in a solvent can be expected to be determined by the energetic cost of mixing the two components, which in turn can be evaluated through their enthalpy of mixing [35]. When the solute is a nanostructured material, as in the present case, where interaction with the solvent takes place on its surface, the most intuitive and appropriate way of deriving the enthalpy of mixing is probably that based on the analysis of surface energies. More specifically, for a two-dimensional (2D) material dispersed in a solvent in the form of thin platelets, the enthalpy of mixing per unit volume of solvent $\left(\Delta \overline{\mathrm{H}}_{\text {mix }}\right)$ can be written to a first approximation as [5]

$$
\Delta \overline{\mathrm{H}}_{\text {mix }}=\frac{2}{\mathrm{t}}\left(\delta_{2 D}-\delta_{\text {sol }}\right)^{2} \varphi
$$

, where $t$ is the platelet thickness, $\phi$ is the volume fraction of the $2 \mathrm{D}$ material in the solvent and $\delta_{2 \mathrm{D}}=\sqrt{\mathrm{E}_{\text {surf }}^{2 \mathrm{D}}}\left(\delta_{\text {sol }}=\sqrt{\mathrm{E}_{\text {surf }}^{\text {sol }}}\right)$ is the square root of the surface energy of the material (solvent). This approach to solubility analysis has been recently shown to be equivalent to that based on the cohesive energy density of solvent and solute [36]. Specifically, when only their total cohesive energy densities are considered, the enthalpy of mixing is given by [37]

$$
\Delta \overline{\mathrm{H}}_{\text {mix }}=\left(\delta_{T, 2 D}-\delta_{T, s o l}\right)^{2} \varphi(1-\varphi)
$$

, with $\phi$ having the same meaning as in Eq. (1) and $\delta_{\mathrm{T}, 2 \mathrm{D}}\left(\delta_{\mathrm{T}, \mathrm{sol}}\right)$ being the Hildebrand solubility parameter of the 2D material (solvent), which equals the square root of its total cohesive energy density. We note that the analysis based on cohesive energy densities was originally developed for molecular solutes and is indeed ideally suited to such type of solute entities [35]. Nevertheless, in recent years it has also been put to good use to understand and predict the dispersion behavior in solvents of different types of nanostructured materials, including pristine graphene [18], carbon nanotubes [38,39] and nanofibers [32], as well as several inorganic graphene analogues [37].

Eqs. (1) and (2) predict that the energetic cost of dispersing a $2 \mathrm{D}$ material in a solvent will be minimized, and consequently its dispersed amount should be maximized, for solvents with surface energy and Hildebrand solubility parameter that match those of the $2 \mathrm{D}$ material in question. To see how these predictions compare with the actual dispersion behavior of RGO, we prepared suspensions in a number of solvents that cover a wide range of surface energy values by sonicating and then centrifuging the 
RGO material under identical conditions for all solvents. The amount of RGO retained in the supernatant was estimated by measuring its optical absorbance at $660 \mathrm{~nm}$ (see Experimental Section). Fig. 2a plots the absorbance data for dispersions obtained at a centrifugal force of $500 \mathrm{~g}$ against solvent surface tension. (Note that solvent surface tension equals its surface energy minus an entropy-related term that is approximately constant for all solvents, i.e. $\sim 30 \mathrm{~mJ} \mathrm{~m}^{-2}$ at room temperature [5].) We see that successful solvents of RGO are scattered over a rather broad range of surface tension values (roughly between 20 and $50 \mathrm{~mJ} \mathrm{~m}^{-2}$ ), in contrast to the case of pristine graphene and other nanostructured materials, for which good solvents are usually clustered within a significantly narrower (i.e., $\sim 10-15$ vs. $\sim 30 \mathrm{~mJ} \mathrm{~m}^{-2}$ ) range of values $[18,32,37]$. We can rule out that the origin of such discrepancy is related to the lateral size of the RGO sheets. In principle, the scattering of surface tension values for good solvents should be expected to increase with decreasing lateral size of the 2D sheets [37]. However, the RGO dispersions prepared here in the different solvents were made up of sheets with very similar sizes to those of other $2 \mathrm{D}$ materials that have been investigated previously (i.e., between a few and several hundred nanometers; see below).

Another possibility that could account for the large scattering of surface tension values in RGO would be a non-optimal preparation of the dispersions. More specifically, a centrifugal force of $500 \mathrm{~g}$ might not be sufficient to provide proper discrimination between good and poor solvents. Therefore, we prepared suspensions at higher centrifugal forces, namely, 1000 and $2000 \mathrm{~g}$. While the results obtained at $1000 \mathrm{~g}$ were much like those of Fig. 2a, some differences were observed for the samples prepared at $2000 \mathrm{~g}$, as shown in Fig. 2b. (Absorbance data for RGO dispersions in all solvents at 500 and $2000 \mathrm{~g}$ are given in Table S1 of the Supplementary content together with solvent surface tension and solubility parameters taken from the literature [35,40].) In the latter case, it can be seen that the dispersed amount displays two distinct peaks at about 20-25 and 45-50 $\mathrm{mJ} \mathrm{m}^{-2}$, which again is different to what is usually observed for other nanostructured materials. Fig. $2 \mathrm{c}$ shows a digital photograph of a representative selection of the suspensions $(2000 \mathrm{~g})$ arranged from left to right in increasing order of solvent surface tension. We note that the most successful solvents, both in the 20-25 and 45-50 $\mathrm{mJ} \mathrm{m}^{-2}$ range, tended to be alcohols, e.g. ethanol, 1-butanol, ethylene glycol or ethanolamine, although solvents such as $\gamma$-butyrolactone or $N, N$-dimethylformamide (DMF) also exhibited a reasonably good performance. As for the long-term stability of these RGO dispersions, those prepared in the best solvents identified (e. g., isopropyl 
alcohol, ethanol, 1-butanol and even DMF) were remarkably stable, the amount of sediment developed after more than three months being undetectable to the naked eye. The RGO dispersions in comparatively poorer solvents, such as dimethyl sulfoxide or acetonitrile, were less stable, visible sediment being developed over the course of weeks. This type of result is consistent with what was previously found for dispersions of pristine graphene [18] and platelet-type graphite nanofibers (PGNFs) [32]. These RGO dispersions in organic solvents were mostly constituted by individual, single-layer sheets, as exemplified in the AFM images of Fig. 3a and b for dispersions in ethanol and DMF, respectively. Furthermore, the sheets exhibited lateral dimensions that were similar to those of the as-prepared RGO product (Fig. 1d).

Rather than being due to some artifact in the measurements, we believe that the previous results are essentially a reflection of the peculiar structure of RGO, and as a whole can be interpreted accordingly. We note that a material with a surface that is structurally and chemically homogeneous (e.g. pristine graphene) should possess a welldefined surface energy value that is locally uniform across its entire surface. It is therefore not surprising that for such a kind of material, successful solvents are located within a rather narrow range of surface energy (or surface tension) values. On the other hand, RGO sheets are composed of two chemically differentiated types of nanometerscale domain, namely, pristine and highly oxidized graphene domains [19-21], which should be associated to different values of surface energy. Although in this case the dispersion behavior might be described by a single global (or effective) surface energy value, so that the enthalpy of mixing for RGO would also be given by Eq. (1), a more insightful analysis could be probably attained by explicitly considering the contributions of each type of domain to the enthalpy of mixing. In a formal and probably quite simplified treatment, we can assume that a fraction $\eta_{\mathrm{pr}}$ of the RGO surface is made up of pristine graphene domains with surface energy $E_{\text {surf }}^{\text {pr }}$ and a fraction $\eta_{\text {ox }}$ consists of highly oxidized domains with surface energy $E_{\text {surf }}^{o x}$ with $\eta_{p r}+\eta_{o x}=1$. Then, by considering the balance of all the possible intermolecular interactions in a way analogous to that followed in the derivation of Eq. (1), the enthalpy of mixing for RGO can be derived as:

$$
\Delta H_{m i x}=\frac{2}{\mathrm{t}}\left[\eta_{p r}\left(\delta_{p r}-\delta_{s o l}\right)^{2}+\eta_{o x}\left(\delta_{o x}-\delta_{s o l}\right)^{2}\right] \varphi
$$


, where $\delta_{\mathrm{pr}}=\sqrt{\mathrm{E}_{\mathrm{surf}}^{\mathrm{pr}}}, \delta_{\mathrm{ox}}=\sqrt{\mathrm{E}_{\text {surf }}^{\mathrm{ox}}}$, and $t, \phi$ and $\delta_{\mathrm{sol}}$ have the same meaning as in Eq. (1). Minimization of Eq. (3) indicates that dispersion of RGO should be most favored in those solvents that satisfy the following relation:

$\delta_{\mathrm{sol}}=\eta_{\mathrm{pr}} \delta_{\mathrm{pr}}+\eta_{\mathrm{ox}} \delta_{\mathrm{ox}}$

, or equivalently, in solvents with surface energy values in-between those of the pristine and oxidized domains of RGO, the actual value being dictated by the specific weight of each type of domain (i.e., $\eta_{\mathrm{pr}}$ and $\eta_{\mathrm{ox}}$ ). A similar reasoning could be applied to the analysis based on total cohesive energy densities, in such a way that the most successful solvents for dispersing RGO would be mathematically those with Hildebrand solubility parameter given by a linear combination of the corresponding parameters for the pristine and oxidized domains of RGO, with the $\eta_{\text {pr }}$ and $\eta_{\text {ox }}$ values as the weighting factors. Although, as mentioned before, there is some nitrogen present in RGO, in describing our simple model we have only mentioned the main heteroatom present, i. e., oxygen. However, we could easily incorporate all other functionalities in our model just by considering pristine and non-pristine domains, the latter containing oxygen and/or nitrogen atoms, and the results of our simplified treatment would be equivalent. Here, we discuss the results as a function of the majority heteroatom for the sake of simplicity.

It should be stressed that the assumptions made to derive Eq. (3) may not accurately capture the complexity of the processes that drive the dispersion behavior of RGO. For example, interaction of the solvent molecules with the RGO surface at the boundary regions between the pristine and highly oxidized graphene domains might be different to those taking place inside each type of domain. Considering that the domains are typically just a few nanometers in lateral size $[19,20,34]$, the contribution of the boundary regions to the interaction of RGO sheets with their surrounding solvent could be quite significant. However, at the very least Eqs. (3) and (4) suggest that the dispersibility of RGO should be influenced by, and thus contain elements from, the dispersion behavior of each type of domain. For pristine graphene, a surface energy of $\sim 70-80 \mathrm{~mJ} \mathrm{~m}^{-2}$ has been previously determined, and accordingly its most successful solvents have been shown to possess surface tensions in the $40-50 \mathrm{~mJ} \mathrm{~m}^{-2}$ range [5]. Thus, we can conclude that many solvents with surface tension in such a range are reasonably good solvents of RGO (as noted in Fig. 2a and b) because they exhibit an optimal interaction with its pristine graphene domains. On the other hand, to the best of our knowledge no direct estimates for the surface energy of the highly oxidized domains 
of RGO are available. However, they should be chemically very similar to heavily oxidized edge planes of graphitic structures. For the latter, the surface energy has been experimentally estimated to be about $15 \mathrm{~mJ} \mathrm{~m}^{-2}$ lower than that of the pristine basal plane of graphite [41]. In agreement with this, we have recently demonstrated that the best solvents for PGNFs, which are a type of graphitic nanostructure with a surface comprised of a very high density of oxidized carbon atoms in edge-plane configuration, are those with surface tensions approximately between 20 and $35 \mathrm{~mJ} \mathrm{~m}^{-2}$ [32]. Significantly, a large fraction of the best solvents for RGO were seen to fall within this range (Fig. 2a and b), so we infer that such solvents display an optimal interaction with the oxidized domains of RGO. Hence, if we assume that the surface energy of the pristine graphene domains of RGO is $\sim 70-80 \mathrm{~mJ} \mathrm{~m}^{-2}$ and that of their highly oxidized counterparts is about $50-60 \mathrm{~mJ} \mathrm{~m}^{-2}$, then according to Eqs. (3) and (4) the best solvents for RGO should possess surface energies in-between (e.g., $\sim 60-70 \mathrm{~mJ} \mathrm{~m}^{-2}$, or equivalently, surface tensions of $\sim 30-40 \mathrm{~mJ} \mathrm{~m}^{-2}$ ). Why this is actually not observed in the experiments (Fig. 2) is not completely clear, but some possible explanation will be given below in the light of further results.

Fig. 4a plots the absorbance data for dispersions prepared at $2000 \mathrm{~g}$ as a function of the Hildebrand solubility parameter, $\delta_{\mathrm{T}}$, of the solvents. Successful solvents are mostly constricted to a narrow range of $\delta_{\mathrm{T}}$ values around $23-25 \mathrm{MPa}^{1 / 2}$, which is similar to what has been observed for pristine graphene. We note that the Hildebrand solubility parameters for good solvents of both pristine graphene and PGNFs (the latter of which can be taken as a reference system for the oxidized domains of RGO) were essentially identical ( 22-24 $\mathrm{MPa}^{1 / 2}$ ), even though the specific solvents were different in each case $[18,32]$. Therefore, it is not surprising that the plot of Fig. 4a exhibits relatively little scattering and is also centered around 23-25 $\mathrm{MPa}^{1 / 2}$. More detailed information can be attained by breaking down the total cohesive energy density on which the Hildebrand parameter is based into its well-known dispersive, polar and hydrogen-bonding components, so that the dispersibility analysis can be made in terms of Hansen solubility parameters [35]. In this case, the enthalpy of mixing of Eq. (2) can be rewritten as

$$
\Delta \overline{\mathrm{H}}_{\text {mix }}=\left[\left(\delta_{\mathrm{D}, 2 \mathrm{D}}-\delta_{D, s o l}\right)^{2}+\left(\delta_{P, 2 D}-\delta_{P, s o l}\right)^{2}+\left(\delta_{H, 2 D}-\delta_{H, s o l}\right)^{2}\right] \varphi(1-\varphi)
$$

, with $\delta_{\mathrm{D}, 2 \mathrm{D}}\left(\delta_{\mathrm{D}, \mathrm{sol}}\right), \delta_{\mathrm{P}, 2 \mathrm{D}}\left(\delta_{\mathrm{P}, \text { sol }}\right)$ and $\delta_{\mathrm{H}, 2 \mathrm{D}}\left(\delta_{\mathrm{H}, \mathrm{sol}}\right)$ being respectively the dispersive, polar and hydrogen-bonding Hansen solubility parameters of the $2 \mathrm{D}$ material (solvent). A 
given Hansen solubility parameter equals the square root of the corresponding component of the cohesive energy density. Again, Eq. (5) predicts that the best solvents for dispersing the 2D material will be those with the three Hansen solubility parameters matching their 2D material counterparts. For RGO, and similar to the analysis based on surface energies discussed above, we can expect that contributions from both types of domain to its dispersion behavior will also be reflected in the analysis based on Hansen solubility parameters.

To bring to light these possible contributions, we have plotted in Fig. 4b-d the absorbance data of the RGO suspensions (2000 g) against the three Hansen parameters, $\delta_{\mathrm{D}}(\mathrm{b}), \delta_{\mathrm{P}}(\mathrm{c})$ and $\delta_{\mathrm{H}}(\mathrm{d})$, of the solvents. The dispersed amounts show relatively little scattering when plotted as a function of $\delta_{D}$ and are maximized for values of this Hansen parameter around $16-17 \mathrm{MPa}^{1 / 2}$, which are very close to those previously reported for both pristine graphene $\left(\sim 18 \mathrm{MPa}^{1 / 2}\right)$ and PGNFs $\left(\sim 16-17 \mathrm{MPa}^{1 / 2}\right)[18,32]$. By contrast, a rather broad distribution, from about 5 to $15 \mathrm{MPa}^{1 / 2}$, is observed when charting the data against $\delta_{\mathrm{P}}$. In this case, we believe that this is not necessarily a reflection of the chemical heterogeneity of the RGO sheets, because similarly broad distributions have been documented even for homogeneous materials (e.g., pristine graphene or inorganic graphene analogues) $[18,37]$. In any event, on comparing Fig. 4c with equivalent plots obtained previously not only for pristine graphene but also for PGNFs, it becomes apparent that solvents with substantial values of the polar Hansen parameter (e.g., around $15 \mathrm{MPa}^{1 / 2}$ ) tend to perform better with RGO than with the other two mentioned materials. Although this is not surprising when RGO is compared to the oxygen-free, pristine graphene sheets, the comparison with PGNFs suggests that the oxygen functional groups in RGO may have a slightly more polar or polarizable nature than that of the groups present in the PGNFs.

The contributions of both the pristine and the highly oxidized domains of RGO to its dispersion behavior are most evident when the absorbance data are plotted against $\delta_{\mathrm{H}}$ (Fig. 4d). Successful solvents are clearly clustered in two distinct groups, one group with $\delta_{\mathrm{H}}$ values between 5 and $10 \mathrm{MPa}^{1 / 2}$ and the other in the $\sim 15-25 \mathrm{MPa}^{1 / 2}$ range. Significantly, the best solvents for pristine graphene sheets have been shown to possess hydrogen-bonding parameters within the former range (those with $\delta_{\mathrm{H}}$ values between 15 and $25 \mathrm{MPa}^{1 / 2}$ being quite inefficient) [18], so we interpret that the pristine graphene domains of RGO exhibit an optimal interaction with such type of solvents. Conversely, 
the surface-oxidized PGNFs were poorly dispersed by solvents with $\delta_{\mathrm{H}}$ values between 5 and $10 \mathrm{MPa}^{1 / 2}$ and very efficiently by those between 15 and $20 \mathrm{MPa}^{1 / 2}$ [32], which leads us to conclude that the latter can interact most effectively with the oxidized domains present in RGO. We note that this result is reasonable in that the oxygen functional groups of RGO, which include a large fraction of hydroxyls, should be prone to establish strong hydrogen bonding interactions with suitable molecules and chemical groups.

According to Eq. (5), the RGO sheets should be characterized by a given set of Hansen solubility parameters. However, following the same line of reasoning as that used for the surface energy-based analysis discussed beforehand [Eqs. (3) and (4)], both the pristine and the highly oxidized domains of RGO should possess their own specific set of Hansen parameters. This implies again that optimal solvents for RGO should be those with Hansen parameters amounting to a certain weighted average of the corresponding parameters for the two types of domain. Such weighted averages can be regarded as the effective Hansen solubility parameters of RGO, which in turn can be estimated from the experimental data of Fig. 4 [32]. The following values were obtained: $\left\langle\delta_{\mathrm{D}, \mathrm{RGO}}\right\rangle \approx 16.9 \pm 0.1 \mathrm{MPa}^{1 / 2},\left\langle\delta_{\mathrm{P}, \mathrm{RGO}}\right\rangle \approx 10.7 \pm 0.1 \mathrm{MPa}^{1 / 2}$ and $\left\langle\delta_{\mathrm{H}, \mathrm{RGO}}\right\rangle \approx$ 14.1 $\pm 0.1 \mathrm{MPa}^{1 / 2}$, being very similar to the ones derived previously for PGNFs $\left(\left\langle\delta_{\mathrm{D}}\right) \approx\right.$ 16.4 $\mathrm{MPa}^{1 / 2},\left\langle\delta_{\mathrm{P}}\right\rangle \approx 8.6 \mathrm{MPa}^{1 / 2}$ and $\left\langle\delta_{\mathrm{H}}\right\rangle \approx 14.7 \mathrm{MPa}^{1 / 2}$ [32]) with the exception of a slightly lower $\delta_{\mathrm{P}}$ parameter for the latter. Compared with pristine graphene $\left(\left\langle\delta_{\mathrm{D}}\right) \approx 18.0\right.$ $\mathrm{MPa}^{1 / 2},\left\langle\delta_{\mathrm{P}}\right\rangle \approx 9.3 \mathrm{MPa}^{1 / 2}$ and $\left\langle\delta_{\mathrm{H}}\right\rangle \approx 7.7 \mathrm{MPa}^{1 / 2}$ [18]), the largest difference can be found in the hydrogen-bonding parameter, which should be a consequence of the presence (absence) of oxygen groups in RGO (pristine graphene). Overall, these results suggest that the dispersibility of RGO is dominated to a larger extent by its oxidized domains than it is by the pristine ones.

Every solvent as well as RGO itself can be given a specific position in the so-called Hansen parameter space, which is defined by the three solubility parameters. In principle, the shorter the distance between a given solvent and RGO in the Hansen space, the better the ability of the solvent to disperse RGO should be. Such a distance, R, has been defined by Hansen as [35]

$$
\mathrm{R}=\sqrt{4\left(\delta_{\mathrm{D}, 2 \mathrm{D}}-\delta_{\mathrm{D}, \mathrm{sol}}\right)^{2}+\left(\delta_{\mathrm{P}, 2 \mathrm{D}}-\delta_{\mathrm{P}, \mathrm{sol}}\right)^{2}+\left(\delta_{\mathrm{H}, 2 \mathrm{D}}-\delta_{\mathrm{H}, \mathrm{sol}}\right)^{2}}
$$


, with the different parameters having the same meaning as in Eq. (5). Therefore, if the effective Hansen parameters obtained above for RGO are reasonably correct, we should expect its dispersed amount in the different solvents to increase with decreasing $\mathrm{R}$. The plot shown in Fig. 5a (black dots) indicates that this is essentially the case. Even though we observe significant scattering in the measured absorbance for very similar values of $\mathrm{R}$, which can be ascribed to the fact that the Hansen parameter theory does not capture all the details of the solvent-material interaction, there is a clear trend for the best performing solvents to be those lying closer to the origin of the R axis in the plot. We also note from Fig. 5a that none of the solvents employed in this study was actually very close to RGO in the Hansen parameter space, i.e. very close to the origin of the R axis. In fact, it was difficult to find solvents of relatively common usage that lay exceedingly close to RGO. In the following, we show that this problem could be circumvented by the use of appropriate solvent mixtures.

In principle, the Hansen solubility parameters of a mixture of two solvents are given by a weighted average of the corresponding parameters of the two individual solvents, the volume fraction of each solvent being the weighting factor $[35,42]$. This means that any mixture of these two solvents is located at a certain point along the straight line that connects the two solvents in the Hansen parameter space. As a consequence, mixtures of two solvents could be more effective in dispersing a given nanostructured material than any of the two solvents alone, provided that such mixtures lie closer to the material in question than the solvents themselves. For example, this idea has been successfully applied to the preparation of even highly concentrated suspensions of several inorganic graphene analogues in mixtures of two solvents (water and ethanol) that were highly inefficient when used alone $[42,43]$. In our case, inspection of the Hansen parameters of the different solvents tested with RGO (Table S1 in the Supplementary content) suggested that certain mixtures of ethanol and $N$-methyl-2-pyrrolidone (NMP) should possess solubility parameters nearly identical to those derived for RGO. Consequently, we would expect some ethanol/NMP mixtures to afford larger dispersed amounts of RGO than those attained with any of the two solvents alone, and even with any of the solvents that were used here. To test this hypothesis, we prepared dispersions of RGO in ethanol/NMP mixtures with different compositions and measured their absorbance at $660 \mathrm{~nm}$, the results of which are shown in Fig. 5b. In agreement with the expectations, certain solvent mixtures (e.g., $20 \mathrm{vol} \%$ ethanol/80 vol\% NMP) yielded substantially larger concentrations of RGO than those achieved by either ethanol or NMP. These 
results have been included (red dots) in the plot of Fig. 5a, where we see that they are generally consistent with the trends observed for the single-component solvents, i.e. lower values of R tend to give larger amounts of dispersed RGO. Incidentally, the fact that Hansen solubility parameters of a mixture of two solvents are given by a weighted average of the corresponding parameters of the two individual solvents, makes it clear that the residual $3 \%$ volume fraction of water coming from the starting aqueous RGO slurry that will be present in every tested dispersion will not introduce but a small error in our analysis.

The previous results are significant in that they provide support not only to the general validity of the Hansen parameter theory as a tool to understand and predict the dispersion behavior of RGO both in single-component solvents and solvent mixtures, but also to the ideas developed specifically to analyze the peculiarities of this chemically heterogeneous material [e.g., in connection with Eqs. (3) and (4)]. We note that NMP has been identified as one of the best solvents for pristine graphene, and indeed both the surface energy and the Hansen solubility parameters of NMP and pristine graphene are very similar [18]. Likewise, previous work with PGNFs has revealed that ethanol displays a good interaction with heavily oxidized graphitic surfaces [32], so we can expect both the surface energy and the Hansen parameters of this solvent to be close to those of the highly oxidized domains in RGO. Thus, when we base our analysis on surface energies, if the best solvents for dispersing RGO are defined by Eq. (4), then it immediately follows that appropriate mixtures of ethanol and NMP should possess $\delta_{\text {sol }}$ values that satisfy Eq. (4), and consequently should afford the highest dispersed amounts of RGO. A similar reasoning can be made for an analysis based on Hansen solubility parameters, leading to the same conclusion. Importantly, these ethanol/NMP mixtures yielded the highest dispersed amounts of RGO achieved in this work, ethanol and isopropanol being the best performing single-component solvents (see Table S1 in the Supplementary content). Therefore, if data from ethanol/NMP mixtures were to be included in the plots of Fig. 2, then peaks of dispersed concentrations would be located more or less where Eq. (4) predicts, i.e. in-between good solvents of pristine graphene and highly oxidized graphitic surfaces. We thus conclude that none of the single-component solvents used to prepare the RGO dispersions happened to possess the optimal characteristics that allow maximizing the dispersibility of this chemically heterogeneous material. 
Finally, we note that the dispersion tests described above were carried out for initial concentrations of the RGO product in the solvents of $\sim 0.1 \mathrm{mg} \mathrm{mL}^{-1}$, and hence the concentration of all the resulting colloidal suspensions was necessarily below $0.1 \mathrm{mg}$ $\mathrm{mL}^{-1}$. However, for practical purposes the availability of more concentrated suspensions would be advantageous, for example, with a view to the preparation of composite materials or conductive inks. We explored this question in the case of ethanol, as it was one of the two best single-component solvents identified for RGO. To this end, the asprepared aqueous RGO slurry was first rinsed with ethanol to displace the water molecules and convert it to thick ethanolic slurry. The latter was added to a certain volume of ethanol, treated first in a bath sonicator, and then with a tip sonicator to best favor exfoliation of some agglomerated particles, centrifuged at $500 \mathrm{~g}$ for $5 \mathrm{~min}$, and the concentration of RGO remaining in the supernatant estimated by UV-vis absorption spectroscopy. Significantly, we were able to achieve concentrations between 0.5 and 1 $\mathrm{mg} \mathrm{mL} \mathrm{m}^{-1}$, which facilitated the preparation of RGO-based composite materials. This possibility was exemplified for RGO-ethyl cellulose composites. Typically, ethyl cellulose was dissolved at a concentration of $14 \mathrm{mg} \mathrm{mL}^{-1}$ in a concentrated ethanolic RGO dispersion. The resulting solution was poured onto a poly(tetrafluoroethylene) mold and the solvent was allowed to evaporate under ambient conditions, leaving behind an RGO-ethyl cellulose composite film (Fig. 6a, digital picture of a film with 2 wt\% of RGO loading and pure ethyl cellulose film; b and c, field emission scanning electron microscopy images of the composite fracture surface at two different magnifications). The composite shows a lamellar structure which must be induced by the presence of RGO, as it is absent in pure ethyl cellulose films (images not shown). TGA under air flow indicated that the addition of RGO improves the stability of the polymer towards oxidation (Fig. 6c), as its gasification is shifted to higher temperatures in the presence of the sheets (upshift of $\sim 12-13{ }^{\circ} \mathrm{C}$ for $2 \mathrm{wt} \%$ loading of RGO). This result suggests that the RGO sheets exhibit some barrier effect towards oxygen molecules, providing a certain protection to the polymer against oxidation. More detailed studies of these composites are underway and will be reported elsewhere.

\section{Conclusions}

The dispersibility of reduced graphene oxide (RGO) sheets in solvents was quantitatively investigated and the results could be rationalized on the basis of solvent surface energy and Hansen solubility parameters. In general, the dispersion behavior of 
RGO was noticeably different to that exhibited by pristine graphene, but at the same time it incorporated some attributes that are characteristic of the latter. Such a result was interpreted to reflect the well-known chemical heterogeneity of RGO sheets, with their pristine and heavily oxidized nanometer-sized graphene domains contributing differently to the interaction of the sheets with solvent molecules. Estimates of the effective Hansen solubility parameters for RGO obtained from the experimental data of dispersibility proved to be useful towards the rational design of solvent mixtures that afforded significant increases in the dispersed amounts of the material. Overall, the present work should expedite the liquid-phase processing of RGO for practical uses. For example, highly concentrated RGO suspensions could be achieved with some of the best solvents, which facilitated the preparation of RGO-polymer composites as demonstrated in the case of ethyl cellulose.

\section{Acknowledgements}

Financial support from the Spanish MINECO and the European Regional Development Fund (project MAT2011-26399) is gratefully acknowledged. M.A.-V. is thankful for the receipt of a pre-doctoral contract (FPI) from Spanish MINEC and R.R. thanks the receipt of a pre-doctoral contract (FPU) from the Spanish MECD.

\section{References}

[1] Geim AK, Novoselov KS. The rise of graphene. Nature Mater. 2007; 6:183-91.

[2] Geim AK. Graphene: Status and prospects. Science 2009; 324:1530-4.

[3] Novoselov KS, Fal'ko VI, Colombo L, Gellert PR, Schwab MG, Kim K. A roadmap for graphene. Nature 2012; 490:192-200.

[4] Bonaccorso F, Lombardo A, Hasan T, Sun Z, Colombo L, Ferrari AC, Production and processing of graphene and 2D crystals. Mater. Today 2012;15:564-89.

[5] Hernandez Y, Nicolosi V, Lotya M, Blighe FM, Sun Z, De S, et al. High-yield production of graphene by liquid-phase exfoliation of graphite. Nature Nanotech. 2008; 3:563-8.

[6] Park S, Ruoff RS. Chemical methods for the production of graphenes. Nature Nanotech. 2009; 4:217-24. 
[7] Cai M, Thorpe D, Adamson DH, Schniepp HC. Methods of graphite exfoliation J. Mater. Chem. 2012; 22:24992-5002.

[8] Compton OC, Nguyen ST. Graphene oxide, highly reduced graphene oxide, and graphene: versatile building blocks for carbon-based materials. Small 2010; 6:711-23.

[9] Quintana M, Vazquez E, Prato M. Organic functionalization of graphene in dispersions. Acc. Chem. Res. 2013; 46:138-48.

[10] Lotya M, Hernandez Y, King PJ, Smith RJ, Nicolosi V, Karlsson LS, et al. Liquid phase production of graphene by exfoliation of graphite in surfactant/water solutions. J. Am. Chem. Soc. 2009;131:3611-20.

[11] Laaksonen P, Kainlauri M, Laaksonen T, Shchepetov A, Jiang H, Ahopelto J, et al. Interfacial engineering by proteins - exfoliation and functionalization of graphene by hydrophobins. Angew. Chem. Int. Ed. 2010; 49:4946-9.

[12] Guardia L, Fernández-Merino MJ, Paredes JI, Solís-Fernández P, Villar-Rodil S, Martínez-Alonso A, et al. High-throughput production of pristine graphene in an aqueous dispersion assisted by non-ionic surfactants. Carbon 2011; 49: 1653-62.

[13] Fernández-Merino MJ, Paredes JI, Villar-Rodil S, Guardia L, Solís-Fernández P, Salinas-Torres D, et al. Investigating the influence of surfactants on the stabilization of aqueous reduced graphene oxide dispersions and the characteristics of their composite films. Carbon 2012; 50:3184-94.

[14] Sham AYW, Notley SM. A review of fundamental properties and applications of polymer-graphene hybrid materials. Soft Matter, 2013; 9:6645-53.

[15] Du W, Jiang X, Zhu L. From graphite to graphene: direct liquid-phase exfoliation of graphite to produce single- and few-layered pristine graphene. J. Mater. Chem. A 2013; 1:10592-606.

[16] Duch MC, Budinger GRS, Liang YT, Soberanes S, Urich D, Chiarella SE, et al. Minimizing oxidation and stable nanoscale dispersion improves the biocompatibility of graphene in the lung. Nano Lett. 2011;11:5201-7.

[17] Wang Y, Di C, Liu Y, Kajiura H, Ye S, Cao L, et al. Optimizing single-walled carbon nanotube films for applications in electroluminescent devices. Adv. Mater. 2008; 20:4442-9.

[18] Hernandez Y, Lotya M, Rickard D, Bergin SD, Coleman JN. Measurement of multicomponent solubility parameters for graphene facilitates solvent discovery. Langmuir 2010; 26:3208-13. 
[19] Gómez-Navarro C, Meyer JC, Sundaram RS, Chuvilin A, Kurasch S, Burghard M, et al. Atomic structure of reduced graphene oxide. Nano Lett. 2010; 10:1144-8.

[20] Erickson K, Erni R, Lee Z, Alem N, Gannett NW, Zettl A. Determination of the local chemical structure of graphene oxide and reduced graphene oxide. Adv. Mater. 2010; 22:4467-72.

[21] Zhang Q, Zheng H, Geng Z, Jiang S, Ge J, Fan K, et al. The realistic domain structure of as-synthesized graphene oxide from ultrafast spectroscopy. J. Am. Chem. Soc. $2013 ; 135: 12468-74$.

[22] Villar-Rodil S, Paredes JI, Martínez-Alonso A, Tascón JMD. Preparation of graphene dispersions and graphene-polymer composites in organic media. J. Mater. Chem. 2009; 19:3591-3.

[23] Park S, An J, Jung I, Piner RD, An SJ, Li X, et al. Colloidal suspensions of highly reduced graphene oxide in a wide variety of organic solvents. Nano Lett. 2009; 9:15937.

[24] Choi EY, Han TH, Hong JH, Kim JE, Lee SH, Kim HW, Kim SO. Noncovalent functionalization of graphene with end-functional polymers. J Mater Chem. 2010; 20:1907-12.

[25] Loh KP, Bao Q, Eda G, Chhowalla M. Graphene oxide as a chemically tunable platform for optical applications. Nature Chem. 2010; 2:1015-24.

[26] Chen D, Feng H, Li J, Graphene oxide: Preparation, functionalization, and electrochemical applications. Chem. Rev. 2012; 112:6027-53.

[27] Feng, L, Wu L, Qu X. New horizons for diagnostics and therapeutic applications of graphene and graphene oxide. Adv. Mater. 2013; 25:168-86.

[28] Hummers WS, Offeman RE. Preparation of graphitic oxide. J. Am. Chem. Soc. 1958; 80:1339.

[29] Paredes JI, Villar-Rodil S, Solís-Fernández P, Martínez-Alonso A, Tascón JMD. Atomic force and scanning tunneling microscopy imaging of graphene nanosheets derived from graphite oxide. Langmuir 2009; 25:5957-68.

[30] Li D, Müller MB, Gilje S, Kaner RB, Wallace GG. Processable aqueous dispersions of graphene nanosheets. Nature Nanotech. 2008; 3:101-5.

[31] Fernández-Merino MJ, Guardia L, Paredes JI, Villar-Rodil S, Solís-Fernández P, Martínez-Alonso A, et al. Vitamin C is an ideal substitute for hydrazine in the reduction of graphene oxide suspensions. J. Phys. Chem. C 2010; 114:6426-32. 
[32] Guardia L, Paredes JI, Villar-Rodil S, Rouzaud J-N, A. Martínez-Alonso A, Tascón JMD. Discovery of effective solvents for platelet-type graphite nanofibers. Carbon 2013; 53:222-30.

[33] Skoog DA, Holler FJ, Nieman TA, Principles of Instrumental Analysis, Hartcourt Brace \& Company, Philadelphia, 1998.

[34] Joung D, Khondaker SI. Structural evolution of reduced graphene oxide of varying carbon $\mathrm{sp}^{2}$ fractions investigated via Coulomb blockade transport J. Phys. Chem. C $2013 ; 117: 26776-82$

DOI: $10.1021 / \mathrm{jp} 408387 \mathrm{~b}$

[35] Hansen CM, Hansen Solubility Parameters: A User’s Handbook, CRC Press, Boca Raton, 2007.

[36] Hughes JM, Aherne D, Coleman JN. Generalizing solubility parameter theory to apply to one- and two-dimensional solutes and to incorporate dipolar interactions. J. Appl. Polym. Sci. 2013; 130:4483-91.

[37] Cunningham G, Lotya M, Cucinotta CS, Sanvito S, Bergin SD, Menzel R, et al. Solvent exfoliation of transition metal dichalcogenides: dispersibility of exfoliated nanosheets varies only weakly between compounds. ACS Nano 2012; 6:3468-80.

[38] Detriche S, Zorzini G, Colomer JF, Fonseca A, Nagy JB. Application of the Hansen solubility parameters theory to carbon nanotubes. J. Nanosci Nanotechnol. 2008; 8:6082-92.

[39] Bergin SD, Sun Z, Rickard D, Streich PV, Hamilton JP, Coleman JN. Multicomponent solubility parameters for single-walled carbon nanotube-solvent mixtures. ACS Nano 2009; 3:2340-50.

[40] Lide DR (Ed.). CRC Handbook of Chemistry and Physics. $86^{\text {th }}$ ed. Boca Raton: CRC Press; 2005-2006.

[41] Donnet JB, Brendle M, Dhami TL, Bahl OP, Plasma treatment effect on the surface energy of carbon and carbon fibers. Carbon 1986; 24:757-70.

[42] Zhou K-G, Mao N-N, Wang H-X, Peng Y, Zhang H-L. A mixed-solvent strategy for efficient exfoliation of inorganic graphene analogues. Angew. Chem. Int. Ed. 2011; 50:10839-42.

[43] Yao Y, Tolentino L, Yang Z, Song X, Zhang W, Chen Y, et al. High-concentration aqueous dispersions of $\mathrm{MoS}_{2}$. Adv. Funct. Mater. 2013; 23:3577-83. 


\section{Figure 1}
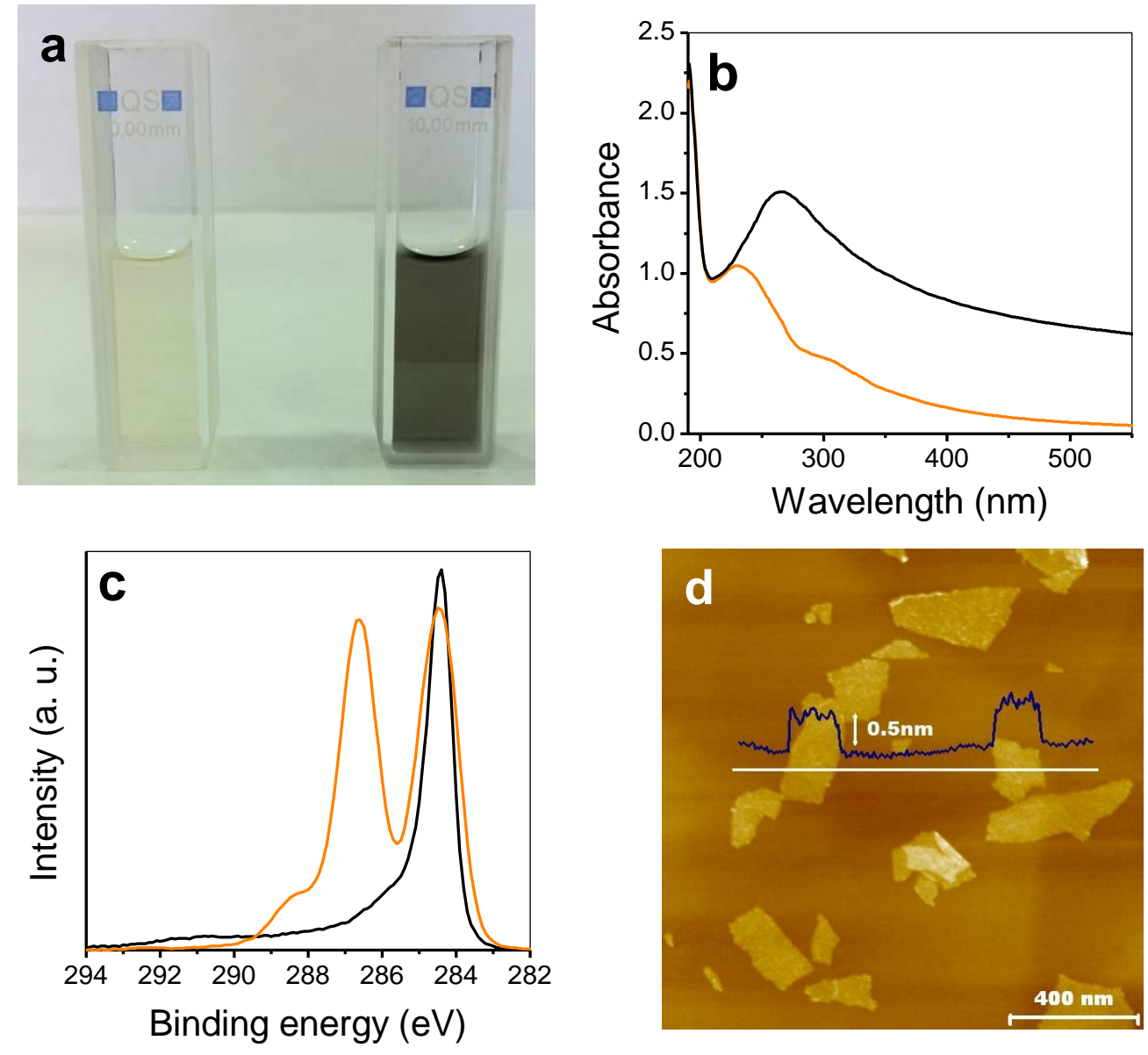

Figure 1. (a) Digital picture of the starting aqueous GO dispersion diluted by a factor of 30 to about $0.1 \mathrm{mg} \mathrm{mL}^{-1}$ (left vial) and the same dispersion after reduction with hydrazine monohydrate and dilution by the same factor (right vial). (b) UV-vis absorption spectra of the aqueous dispersions in (a): GO (orange plot) and RGO (black plot). (c) High resolution C1s XPS spectra of died, drop-casted films of the same dispersions: GO (orange plot) and RGO (black plot). (d) Representative AFM image of the RGO dispersion deposited onto HOPG. A representative line profile (blue line) taken along the marked white line is shown superimposed on the image. 


\section{Figure 2}
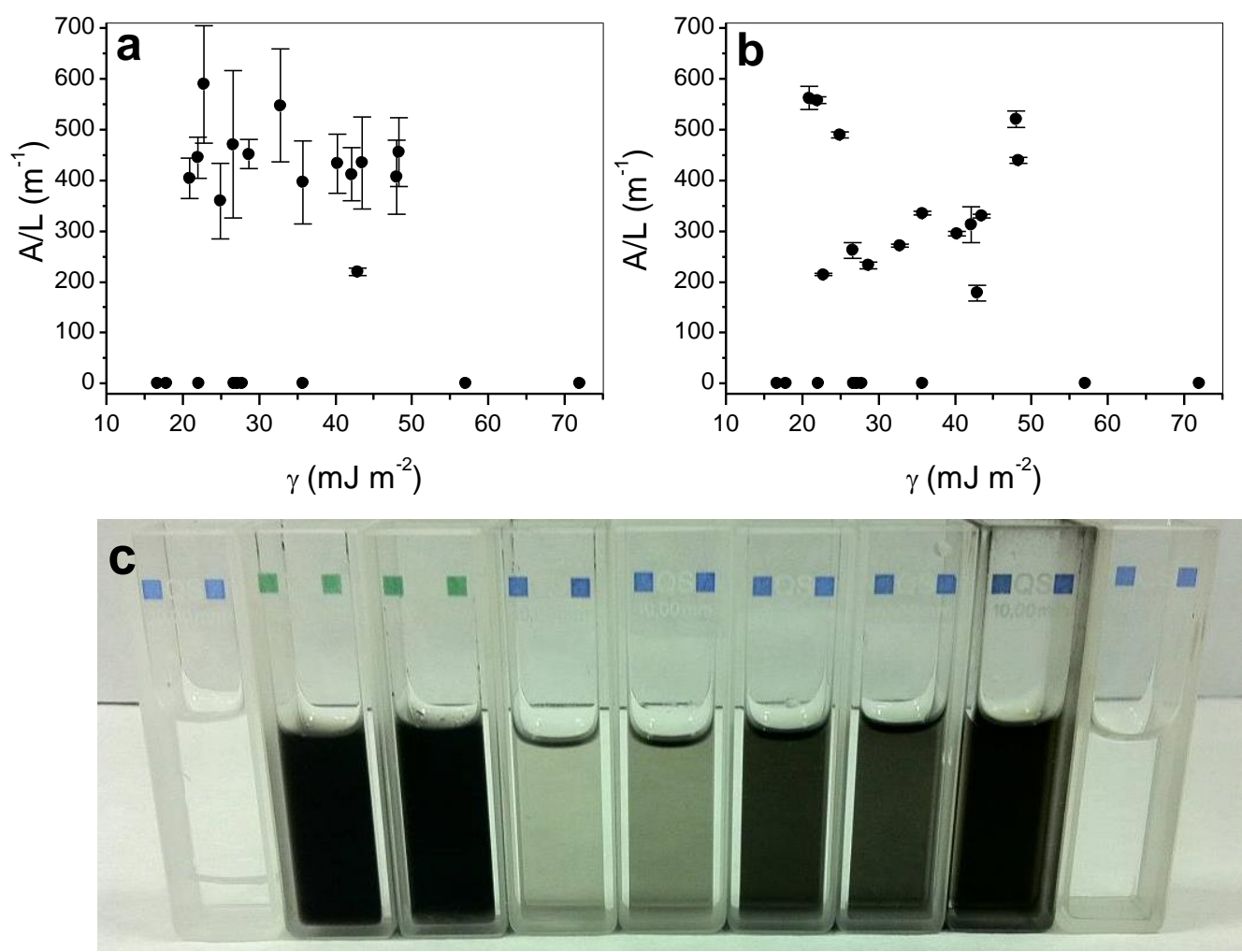

Figure 2. Absorbance at $\lambda=660 \mathrm{~nm}$ per optical path length unit for RGO dispersions obtained at a centrifugal force of (a) $500 \mathrm{~g}$ and (b) $2000 \mathrm{~g}$ against solvent surface tension at $25^{\circ} \mathrm{C}$. (c) Digital photograph of suspensions of RGO prepared at $2000 \mathrm{~g}$ in (from left to right): hexane, ethanol, 1-butanol, tetrahydrofuran (THF), cyclopentanone, $N, N$-dimethylformamide (DMF), $\gamma$-butyrolactone, ethanolamine and water. 


\section{Figure 3}

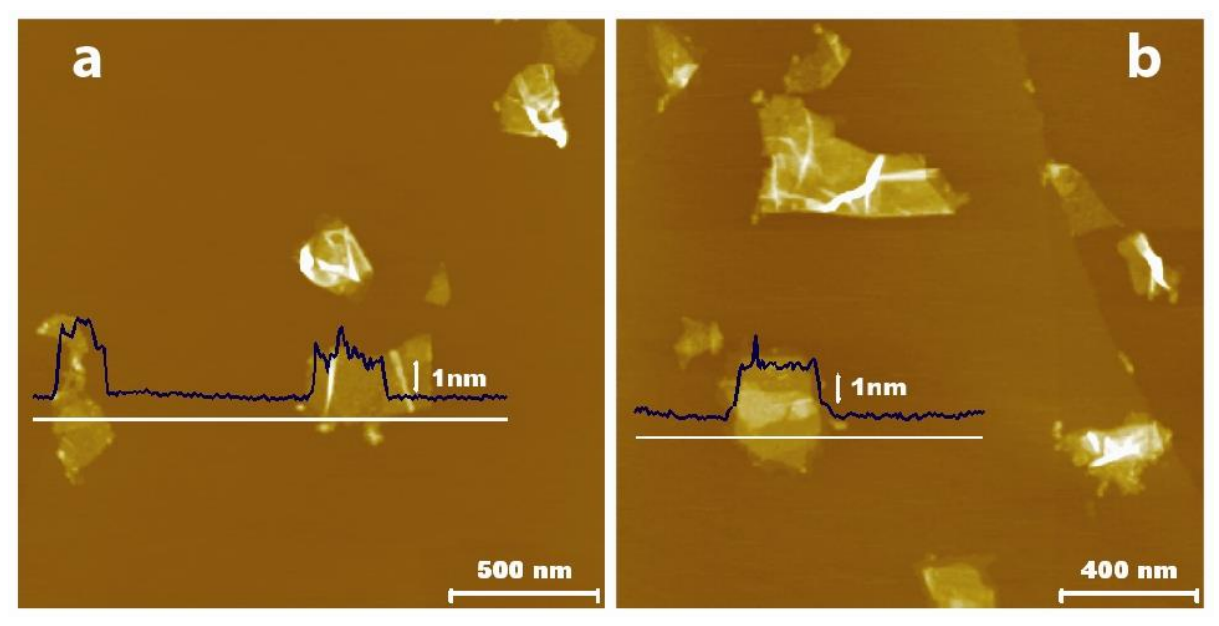

Figure 3. Representative AFM images of RGO dispersions in (a) ethanol and (b) DMF, respectively, deposited onto HOPG substrates. A representative line profile (blue line) taken along the marked white line is shown superimposed on each image. 


\section{Figure 4}
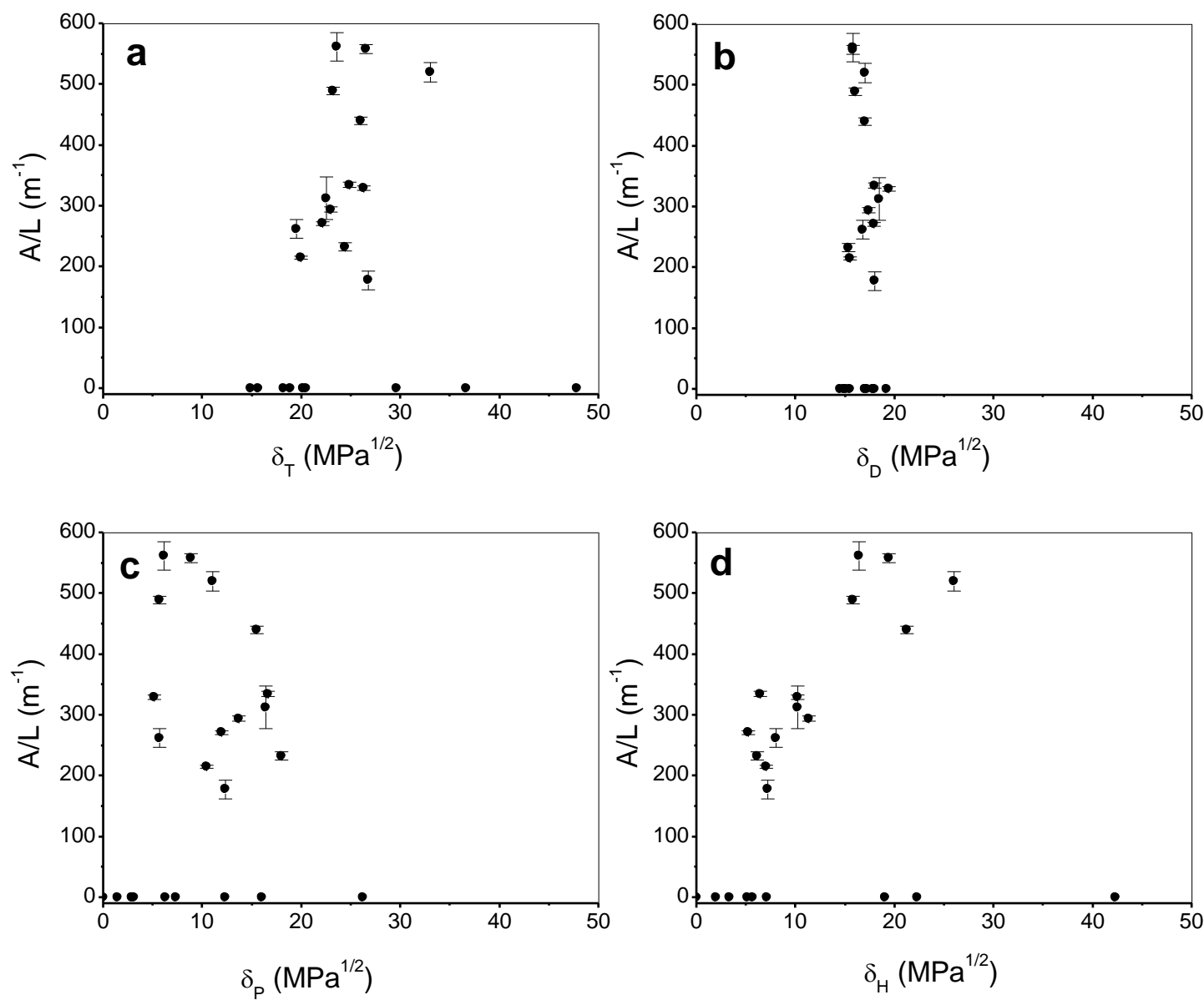

Figure 4. Absorbance at $\lambda=660 \mathrm{~nm}$ per optical path length unit for RGO dispersions prepared at $2000 \mathrm{~g}$ as a function of (a) the Hildebrand solubility parameter, $\delta_{\mathrm{T}}$, and (bd) the three Hansen parameters, $\delta_{\mathrm{D}}(\mathrm{b}), \delta_{\mathrm{P}}(\mathrm{c})$ and $\delta_{\mathrm{H}}(\mathrm{d})$, of the solvents at $25^{\circ} \mathrm{C}$. 


\section{Figure 5}

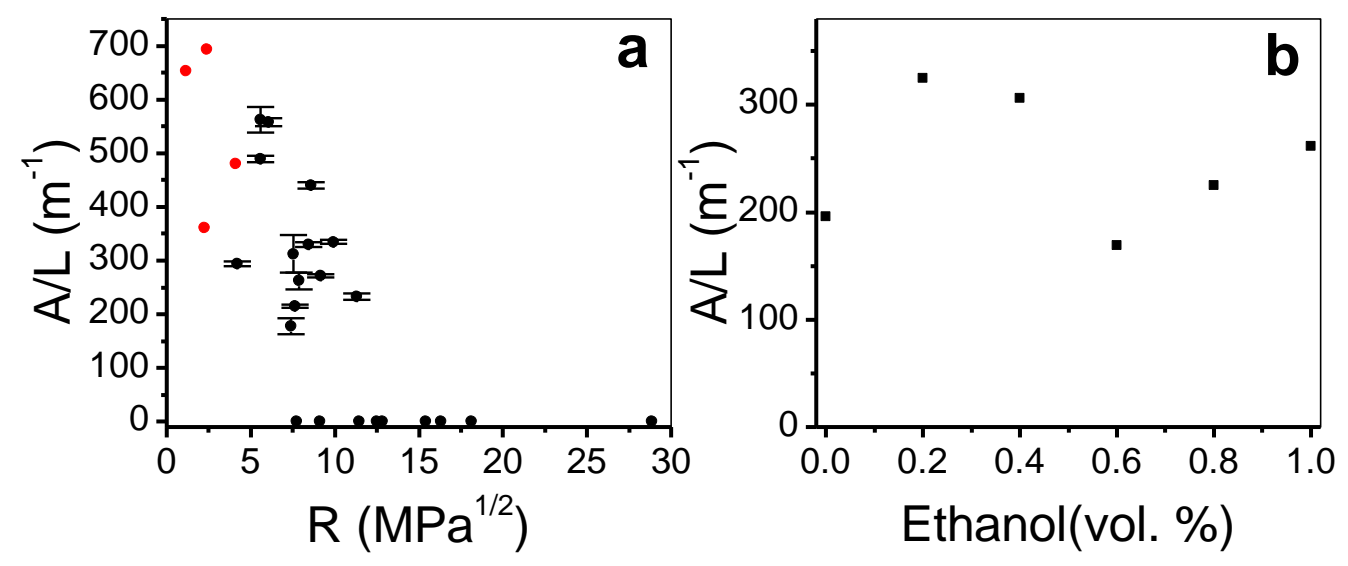

Figure 5. (a) Absorbance at $\lambda=660 \mathrm{~nm}$ per optical path length unit for RGO dispersions prepared at $2000 \mathrm{~g}$ against the distance between the specific solvent and RGO in the Hansen space, R (black dots). The red dots correspond to dispersions of RGO in ethanol/NMP mixtures with different compositions (b) Absorbance at $\lambda=660 \mathrm{~nm}$ per optical path length unit for dispersions of RGO in ethanol/NMP mixtures with different compositions. 


\section{Figure 6}
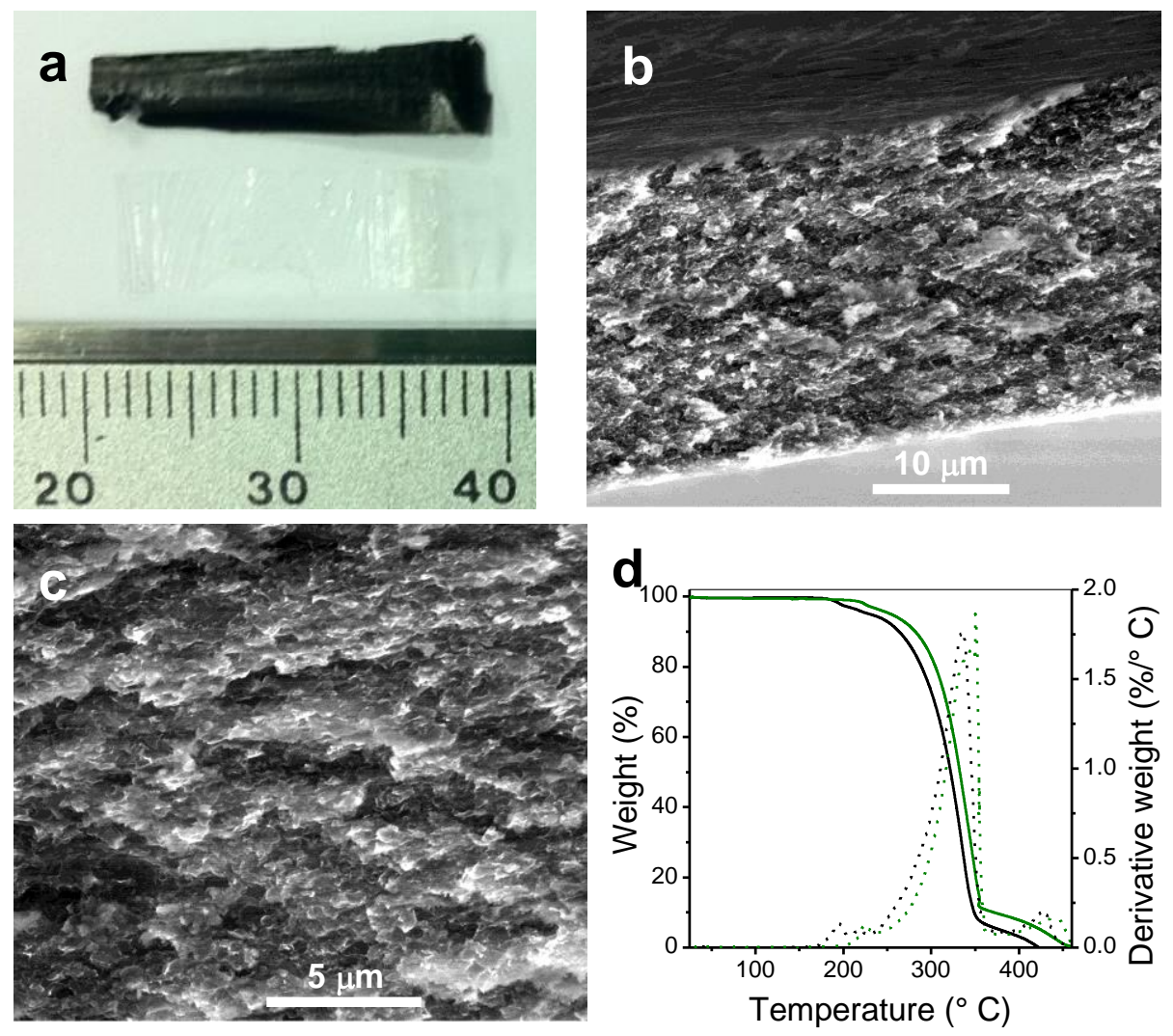

Figure 6. (a) Digital picture of an ethyl cellulose composite film with 2 wt $\%$ of RGO loading (top) and a pure ethyl cellulose film (bottom). T (b,c) Field emission scanning electron microscopy images of the composite fracture surface. (d) Thermogravimetric (solid lines) curves and their derivatives (dotted lines) in air of the composite film (green plot) and of pure ethyl cellulose film (black plot). 